\title{
Nek2-targeted ASO or siRNA pretreatment enhances anticancer drug sensitivity in triple-negative breast cancer cells
}

\author{
JAEHYUNG LEE and LAUREN GOLLAHON \\ Department of Biological Sciences, Texas Tech University, Lubbock, TX, USA
}

Received November 9, 2012; Accepted December 21, 2012

DOI: $10.3892 /$ ijo. 2013.1788

\begin{abstract}
Although the anticancer drugs paclitaxel and doxorubicin are commonly used to treat many solid tumors, their effectiveness is highly variable due to tumor cell resistance. Therefore, it is important to find mechanisms that can be targeted to increase the sensitivity of cancer cells to current chemotherapeutic agents. NIMA-related kinase 2 (Nek2), a serine/threonine kinase is emerging as an important oncogene because of its regulatory role in mitosis. Thus, regulation of the Nek2 expression levels may prove important as a target for cancer treatment. The purpose of our study was to determine whether drug sensitivity was increased in the triple negative breast cancer cell lines MDA-MB-231 and MDA-MB-468 by using small interfering RNA (siRNA) and antisense oligonucleotides (ASOs) against Nek2. To this end, MDA-MB-231 and MDA-MB-468 breast cancer cells transfected with Nek2 siRNA or ASO were exposed to various concentrations of paclitaxel and doxorubicin. Cell viability, cell cycle distribution and apoptosis were evaluated. We observed that drug susceptibility in these transfected cells was dramatically increased compared with either agent alone. FACS results showed that apoptosis was induced in siRNA- and ASO-transfected cells as expected due to the regulatory function of Nek2 in centrosome duplication. Interestingly, the cell cyle was not arrested in transfected cells. We found that siRNA and ASO against Nek2 worked synergistically with paclitaxel and doxorubicin by promoting cell apoptosis. Our results suggest that these drugs in combination with Nek2 siRNA or ASO treatment may improve the sensitivity of cancer cells during chemotherapy treatments.
\end{abstract}

\section{Introduction}

Breast cancer is functionally classified based on molecular profiles. Estrogen receptor (ER), progesterone receptor (PR) and ErbB-2/human epidermal growth factor receptor 2 (HER-2)

Correspondence to: Dr Lauren Gollahon, Texas Tech University, Department of Biological Sciences, PO Box 43131, Lubbock, TX 79409-3131, USA

E-mail: lauren.gollahon@ttu.edu

Key words: NIMA-related kinase 2, small interfering RNA, antisense oligonucleotide, paclitaxel, triple-negative breast cancer status are molecular markers used to determine breast cancer subtypes as well as targets for treatment $(1,2)$. In contrast, triple-negative breast cancer (TNBC) is a breast cancer subtype defined by the lack of expression of ER, PR and HER-2. Treatment of TNBC, which often presents with a more aggressive phenotype, is more difficult due to the paucity of potential target molecules. Therefore, there is a critical need to enhance current systemic treatments and/or identify new targets for the treatment of TNBC (3-5).

Because of the strong correlation between tumor development and specific mutations in the regulatory function of certain cell cycle kinases and cyclin-dependent kinases (CDKs), their potential as targets for anticancer drug design has intensified, with the goal of overcoming the therapeutic challenges presented by TNBC (6-9). Because the centrosome cycle is regulated by protein phosphorylation and given the importance of mitotic and centrosomal kinases, they are attractive targets for anti-mitotic anticancer drugs (10-17).

NIMA-related kinase 2 (Nek2), a serine/threonine centrosomal kinase that is highly expressed and activated during the $\mathrm{S}$ and $\mathrm{G} 2$ phases, is such a target $(18,19)$. Overexpressed Nek2 results in premature centrosome splitting, while centrosomal abnormalities, monopolar spindles and aneuploidy result from overexpression of kinase-dead Nek2 (20,21). Recently, studies have shown that Nek2 expression is elevated in various cancer cell lines, including various breast tumors (22-24). Tsunoda et al (23) demonstrated that Nek2 siRNA could reduce the tumor volume in mouse xenografts. However, combinational studies using Nek2 gene depletion with anticancer drugs have not been reported. In spite of the increasing evidence of the importance of Nek 2 in cancer development, its role in cancer is still far from clear.

In this study, we investigated whether Nek2 depletion by antisense oligonucleotides (ASO) or small interfering RNA (siRNA) against Nek2, promoted drug sensitivity in the TNBC cell lines MDA-MB-231 and MDA-MB-468. Doxorubicin and paclitaxel treated cells were used as positive controls due to the reported problem of low vulnerability (25) with untreated cells as negative controls. Here, we show the effect of Nek2 depletion using siRNA and ASO on two different TNBC cell lines, alone and in combination with paclitaxel and doxorubicin. Alone, siRNA and ASO showed significant reductions in cell viability, mitotic spindle fiber formation and apoptosis. However, in combination with paclitaxel or doxorubicin, Nek2 depletion induced an increase in mitotic abnormalities and apoptosis 
above either silencing alone or anticancer drug treatment alone. Given the difficulty in effectively treating TNBC, our results suggest that either siRNA or ASO targeted against Nek2 may increase TNBC sensitivity to chemotherapy treatments.

\section{Materials and methods}

Cell culture and transfections.MDA-MB-231 and MDA-MB-468 breast cancer cells (ATCC, Manassas, VA) were cultured in Dulbecco's modified Eagle's medium (DMEM) supplemented with 10\% fetal bovine serum (FBS) and penicillin/streptomycin (100 IU $/ \mathrm{ml}$ and $100 \mu \mathrm{g} / \mathrm{ml}$, respectively) under $5 \% \mathrm{CO}_{2}$ in humid conditions at $37^{\circ} \mathrm{C}$. Oligonucleotide transfections were carried out using Lipofectamine 2000 (Invitrogen, Carlsbad, CA) according to the manufacturer's instructions. ASO and siRNA were synthesized by Integrated DNA Technologies Inc., (Coralville, IA). The antisense sequence of phosphorothioate-modified oligodeoxynucleotides against Nek2 was: 5'-GAGCCTGTGCCAATGGTG. The siRNA sequences targeted to Nek2 were 5'-CCAAGGAAAGGCAAUACU UUUdTdT-3' (sense) and 5'-AAGUAUUGCCUUUCCUUG GUUdTdT-3' (antisense). siRNA-A (Santa Cruz Biotechnology, Santa Cruz, CA) was introduced into cells as negative control. Null transfected cells were incubated with Lipofectamine 2000 alone. Cells were collected $24 \mathrm{~h}$ after transfection and analyzed for changes in transcript levels of Nek2. Cells were analyzed $48 \mathrm{~h}$ after transfection, by fluorescence activated cell sorting (FACS) and western blot analysis for protein expression.

Semi quantitative reverse transcriptase PCR (RT-PCR). Total RNA from cells was isolated using RNeasy mini kit (Qiagen, Valencia, CA) following supplier's instructions and treated with DNase I (Promega, Madison, WI) to remove DNA contamination. To generate cDNA from purified total RNA, $1 \mu \mathrm{g}$ of total RNA was added to the SuperScript III first-strand synthesis system reaction mixture according to the manufacturer's (Invitrogen) protocol. Equal amounts of synthesized cDNAs were used to carry out the semi quantitative RT-PCR reactions using the GeneAmp fast PCR Master mix (Applied Biosystems, Carlsbad, CA). The Nek2 primer sequences were: forward, 5'-CCACAGACGAAGTGATGGTG-3'; reverse, 5'-TGATTT TCCCAGCGAGTTCT-3'. Glyceraldehyde-3-phosphate dehydrogenase (GAPDH) was used as control. The primer sequences were: forward, 5'-CACCACCATGGAGAAGGGTG-3'; reverse, 5'-GAGGCATTGCTGTAGATCTTGAGG-3'.

Immunoblotting analysis. Cells were lysed with $20 \mathrm{mmol} / \mathrm{l}$ Tris-HCl (pH 8.0), $137 \mathrm{mmol} / \mathrm{l} \mathrm{NaCl}, 10 \%$ glycerol, $1 \%$ Triton X-100, 2 mmol/l EDTA containing protease and phosphatase inhibitors at $4^{\circ} \mathrm{C}$. A total of $20 \mu \mathrm{g}$ of each lysate was separated by SDS-PAGE and transferred onto a nitrocellulose membrane. Mouse monoclonal antibody against Nek2 (Santa Cruz Biotechnology, 1:1,000), mouse monoclonal anti- $\gamma$-tubulin antibody (Sigma-Aldrich, St. Louis, MO, 1:10,000) and peroxidase-conjugated goat anti-mouse IgG (AnaSpec, Freemont, CA; 1:10,000) were used for immunoblot analyses.

Cell viability assay. The XTT Cell Proliferation Kit (Roche Applied Science, Indianapolis, IN) was used to analyze cell viability. Cells were seeded at 8,000 cells/well into a 96-well culture plate in a final volume of $100 \mu 1$. The XTT mixture (50 $\mu \mathrm{l})$ was added to the wells and incubated for $10 \mathrm{~h}$ at $37^{\circ} \mathrm{C}$. The absorbance of the samples was measured using a Molecular Devices (Sunnyvale, CA) microplate reader at $450 \mathrm{~nm}$ against the reference wavelength of $650 \mathrm{~nm}$. Cell viability assays were carried out at least 3 times independently.

Cell synchronization and FACS. Cells were synchronized by double thymidine. Briefly, after $24 \mathrm{~h}$ incubation, $1 \times 10^{6}$ cells were exposed to $2 \mathrm{mmole} / \mathrm{l}$ of thymidine for $18 \mathrm{~h}$ to presynchronize the cells in $\mathrm{S}$ phase then released by refreshing the medium for $9 \mathrm{~h}$. After release, 2 mmole/l of thymidine was added to block cell cycle for $17 \mathrm{~h}$ followed by fresh DMEM to release cell cycle arrest allowing cells to move forward synchronously throughout G2/M. Cells were harvested 48 h later. Cell cycle distribution was analyzed by FACS. Collected cells were washed in ice-cold PBS, fixed in $70 \%$ ethanol and stored at $-20^{\circ} \mathrm{C}$ until analysis. For FACS, DNA was stained with PBS containing $40 \mu \mathrm{g} / \mathrm{ml}$ of propidium iodide, $100 \mu \mathrm{g} / \mathrm{ml}$ of RNase A, and $0.1 \%$ Triton X-100 for $30 \mathrm{~min}$ at $37^{\circ} \mathrm{C}$. DNA from 10,000 cells was evaluated with a FACSCalibur flow cytometer (Becton-Dickinson, Franklin Lakes, NJ) and cell cycle phases were determined using FCS Express 4.

Immunofluorescence analysis. Cells were fixed in cold methanol and processed for immunocytochemistry. Primary antibodies: mouse monoclonal anti- $\gamma$-tubulin (Sigma-Aldrich, 1:1,000) and mouse monoclonal anti- $\alpha$-tubulin (Abcam, Cambridge, MA; 1:1,000). Secondary antibodies: FITC conjugated goat anti-mouse antibody (Abcam; 1:50) and goat anti-mouse rhodamine conjugated antibody (Upstate, Billerica; MA, 1:500). DNA was counterstained with 4',6-diamidino-2-phenylindole (DAPI). Cells were visualized with an Olympus IX71 inverted deconvolving epifluorescence microscope under 40X using SimplePCI software (Compix).

Apoptosis detection. Apoptosis of treated cells was detected using FITC Annexin V/Dead cell apoptosis kit for flow cytometry (Invitrogen). After staining, fluorescence emission at $530 \mathrm{~nm}$ for Annexin V (FL1) and $>575 \mathrm{~nm}$ for propidium iodide (FL3) was performed using a FACSCalibur flow cytometer (Becton-Dickinson). Data were analyzed using FCS Express 4. Each experiment was performed a minimum of three times to generate statistically relevant results.

\section{Results}

Expression of Nek2 following transfection with Nek2 siRNA or ASO. M231 cells transfected with siRNA and ASO showed gradual loss of Nek2 mRNA expression (Fig. 1). In M468 cells, high concentrations of siRNA demonstrated significantly decreased mRNA expression (Fig. 1D). With ASO transfection, Nek2 mRNA expression declined noticeably even at $5 \mathrm{nM}$ compared with 5 and $10 \mathrm{nM}$ siRNA in M468 cells with higher ASO concentrations suppressing Nek 2 mRNA expression very effectively (Fig. 1E).

The next step was to determine whether Nek2 depletion attenuated protein production or if expression quickly recovered. Therefore, effects of ASO or siRNA against Nek2 protein expression were analyzed $48 \mathrm{~h}$ post-transfection. As shown in 
MDA-MB-231

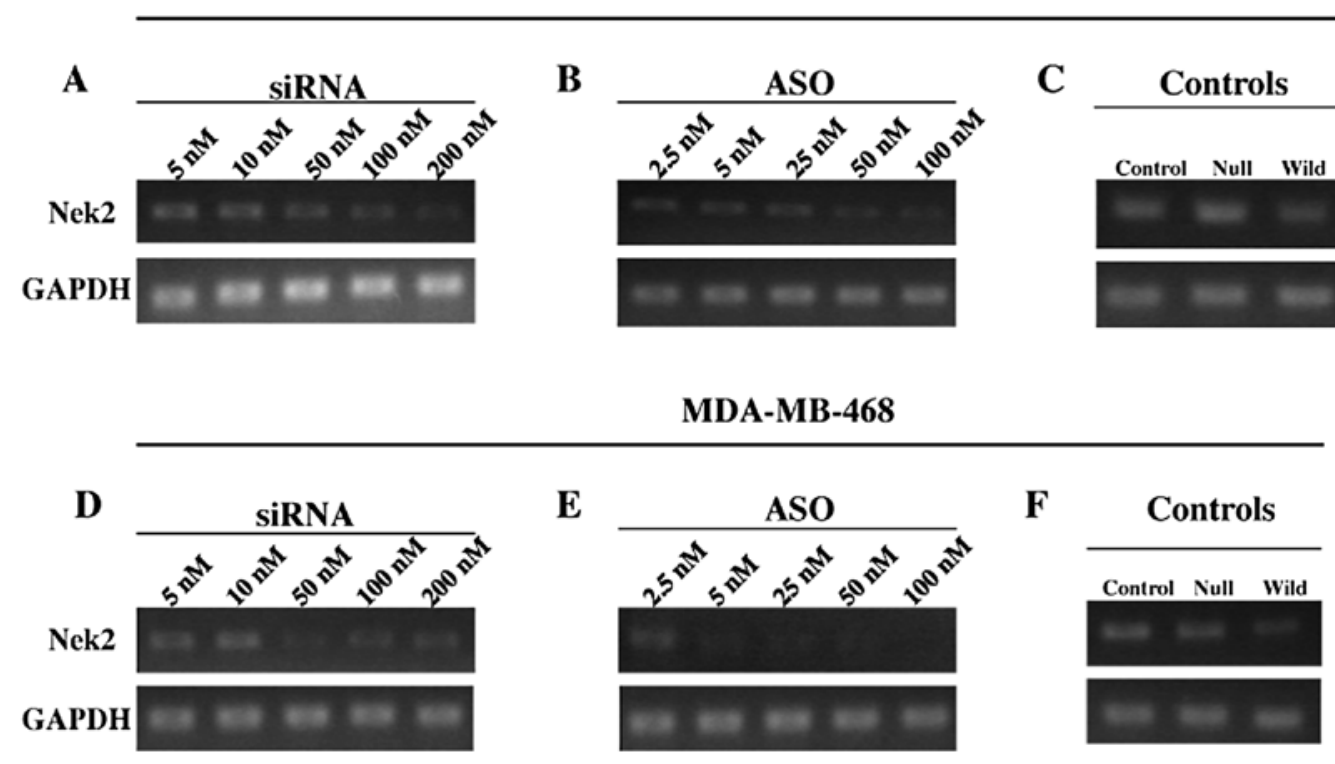

Figure 1. Representative semiquantitative RT-PCR of siRNA or ASO transfection against Nek2 in M231 and M468 triple negative breast cancer cells. RT-PCR analysis of M231 and M468 cells 24 h post-transfection with (A and D) 5, 10, 50, 100 or $200 \mathrm{nM}$ siRNA against Nek2 and (B and E) 2.5, 5, 25, 50 and 100 nM ASO against Nek2, respectively. Each representative gel shows the Nek2 gene product (upper) and GAPDH for control (below). Transfection controls for (C) M231 and (F) M468 were analyzed using control siRNA transfection reagents, null transfection with Lipofectamine 2000 alone, and wild-type, respectively. Each experiment was conducted and analyzed from 3 independent trials.

A
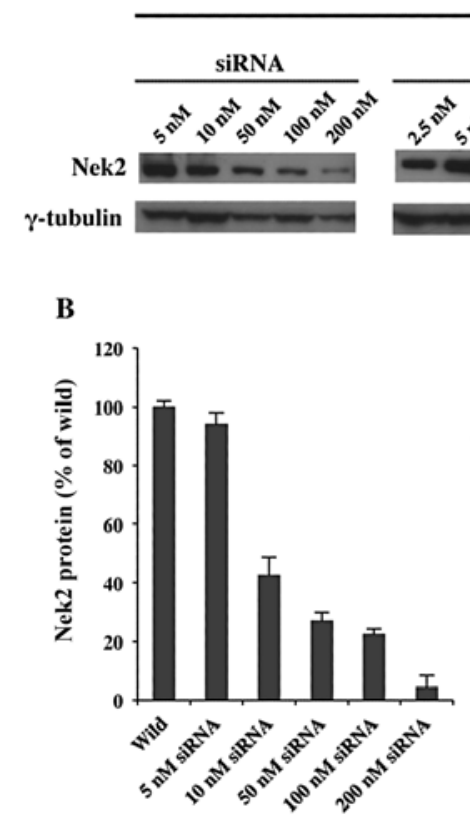

MDA-MB-231

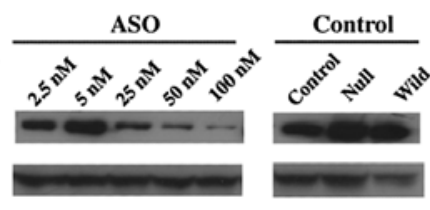

C

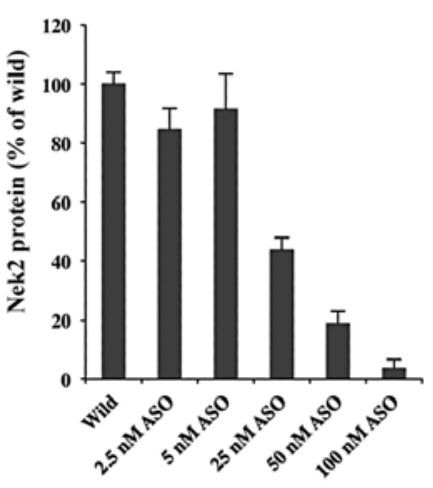

D
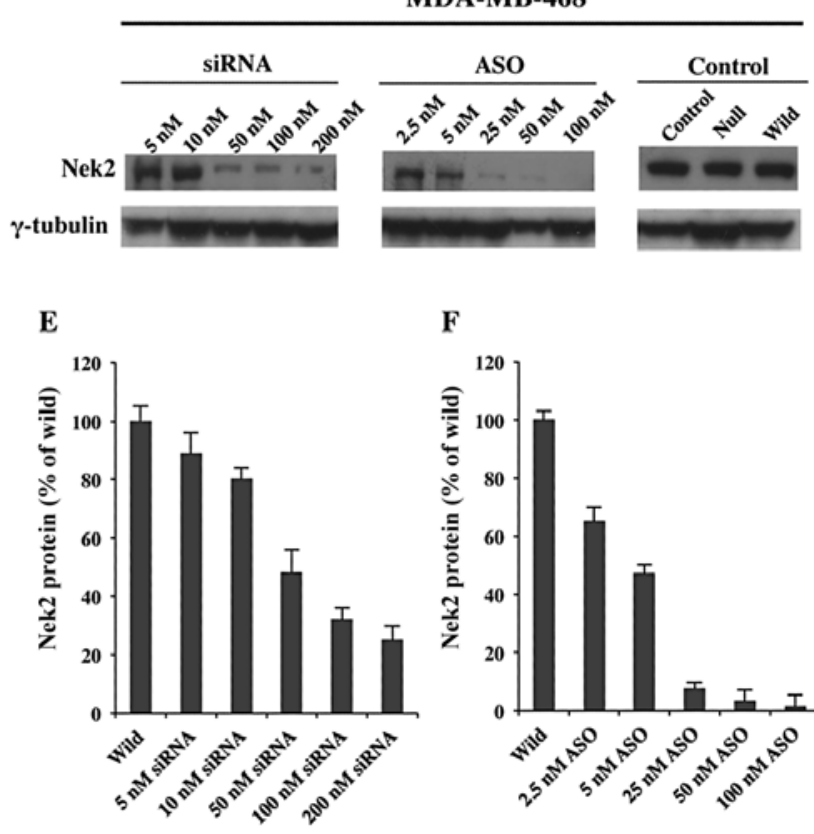

Figure 2. Protein expression levels of Nek2 siRNA or ASO transfected M231 and M468 TNBC cells analyzed by western blot analysis. (A and D) Nek2 protein expression after 48-h transfection in M231 and M468 cells, respectively, with 5, 10,50, 100 and $200 \mathrm{nM}$ of Nek2 siRNA (left) or 2.5, 5, 25, 50 and 100 nM of Nek2 ASO (center). Control cells were treated with control siRNA, Lipofectamine 2000 alone or untreated (right). $\gamma$-tubulin was used as the loading control for each cell line. The bar graphs represent Nek2 protein expression levels with different concentrations of (B and E) siRNA or (C and F) ASO. Nek2 protein expression is given as a percentage standardized against Nek2 expression levels in wild-type cells. Average of three independent experiments and standard deviations are shown ( $\mathrm{n}=3$; error bar, standard deviations).

Fig. 2, Nek2 protein expression was significantly reduced at concentrations of $50 \mathrm{nM}$ siRNA (Fig. 2B). Similarly, higher concentrations of ASO (25 to $100 \mathrm{nM}$ ) showed a significant decrease in Nek2 expression (Fig. 2C).
M468 siRNA and ASO treatments (Fig. 2D-F) also demonstrated overall downregulation of Nek2 protein expression. Higher siRNA concentration results were not as dramatic as those observed for ASO. However, protein expression was still 

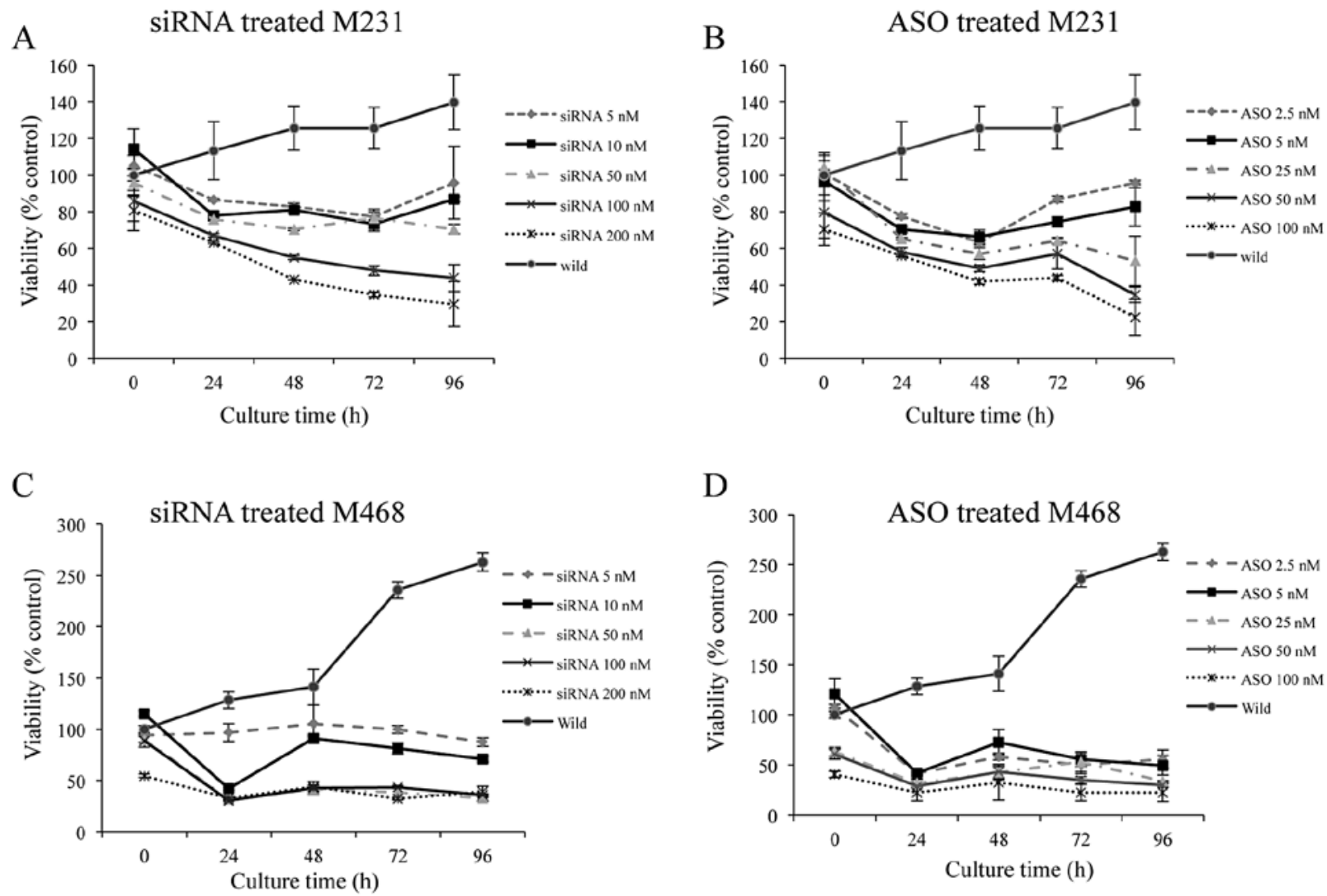

Figure 3. Cell viability of Nek2 siRNA or ASO transfected M231 and M468 breast cancer cells analyzed using the XTT assay. Effect of 5, 10,50, 100 and 200 nM of Nek2 siRNA in (A) M231 and (C) M468; and 2.5, 5, 25, 50 and $100 \mathrm{nM}$ of Nek2 ASO in (B) M231 and (D) M468. Percentage of cell viability is standardized against Nek2 expression levels in untreated (wild-type) cells at $0 \mathrm{~h}$ ( $\mathrm{n}=3$; error bar, standard deviations). Cells were seeded at 8,000 cells/well into a 96 -well culture plate in a final volume of $100 \mu \mathrm{l}$. For all analyses, cell viabilities were standardized against the viability of the non-transfected cells at $0 \mathrm{~h}$. Each time point indicates the time at the addition of $50 \mu \mathrm{l}$ of XTT. The sampling measurement was recorded $10 \mathrm{~h}$ later. Measurements were performed in triplicate for three independent experiments.

markedly reduced. ASO treatment in M486 cells exhibited a more profound effect when administered at higher concentrations (Fig. 2F). Analyses confirmed that Nek2 transcription and translation was successfully depleted by both siRNA and ASO and that the level of depletion was dependent upon concentration.

Cell viability of Nek2 depleted cells. Viability of siRNA transfected M231 cells decreased continuously from 24 to $72 \mathrm{~h}$. After $96 \mathrm{~h}$ post-transfection, viability levels plateaued except in 5 and $10 \mathrm{nM}$ siRNA transfected cells (Fig. 3A). Similarly, cell viability for ASO-transfected M231 cells demonstrated continuous decrease to $72 \mathrm{~h}$ with concentrations of $25 \mathrm{nM}$ and greater showing no significant changes at $96 \mathrm{~h}$ (Fig. 3B).

Transfection of M468 cells with increasing concentrations of siRNA or ASO reduced cell viabilities more effectively (Fig. 3C and D) compared to M231 cells (Fig. 3A and B). Cell viability of M468 cells transfected with $5 \mathrm{nM}$ siRNA showed no change in Nek2 expression. However, with the addition of 50-200 nM siRNA, cell viability significantly decreased after $24 \mathrm{~h}$ (down to 32\%) and remained low (33\%) through 96 h (Fig. 3C).

M468 cells transfected with Nek2-ASO demonstrated significantly reduced viabilities (between 55 to 22\%) for all concentrations (Fig. 3D). Based on these results, $50 \mathrm{nM}$ siRNA and $25 \mathrm{nM}$ ASO were chosen as the optimal concentrations for both cell lines in the subsequent combinatorial studies of siRNA or ASO pretreatment before antitumor agent application. Cell viability of the wild-type (untreated cells) at time $0 \mathrm{~h}$ was normalized as $100 \%$. Cell viability fluctuations for treated samples at time $0 \mathrm{~h}$ were most likely due to the effects of Nek2 depletion on cell proliferation during the $10-\mathrm{h}$ XTT reaction time. Cell viability observed over $100 \%$ in the wild-type control was attributed to continued cell proliferation and metabolism through subsequent time points.

Nek2 is crucial for mitotic spindle formation. In order to determine the effects of silenced Nek 2 on microtubules and spindle pole formation, immunofluorescence microscopy was used to visualize mitotic spindle structural changes. Upon release from cell cycle arrest, disrupted mitoses were observed in both siRNA and ASO transfected cells (Fig. 4A and B) whereas untreated cells exhibited no mitotic deformation. Both cell lines with silenced Nek 2 demonstrated diffuse spindle poles (Fig. 4A and B, gray images) and malformed microtubules (Fig. 4A and B, green images). In addition, $\alpha$-tubulin signal intensities in the spindle microtubules for transfected cells were substantially reduced compared with controls. Similar spindle structures in both Nek2 depleted cell 
A

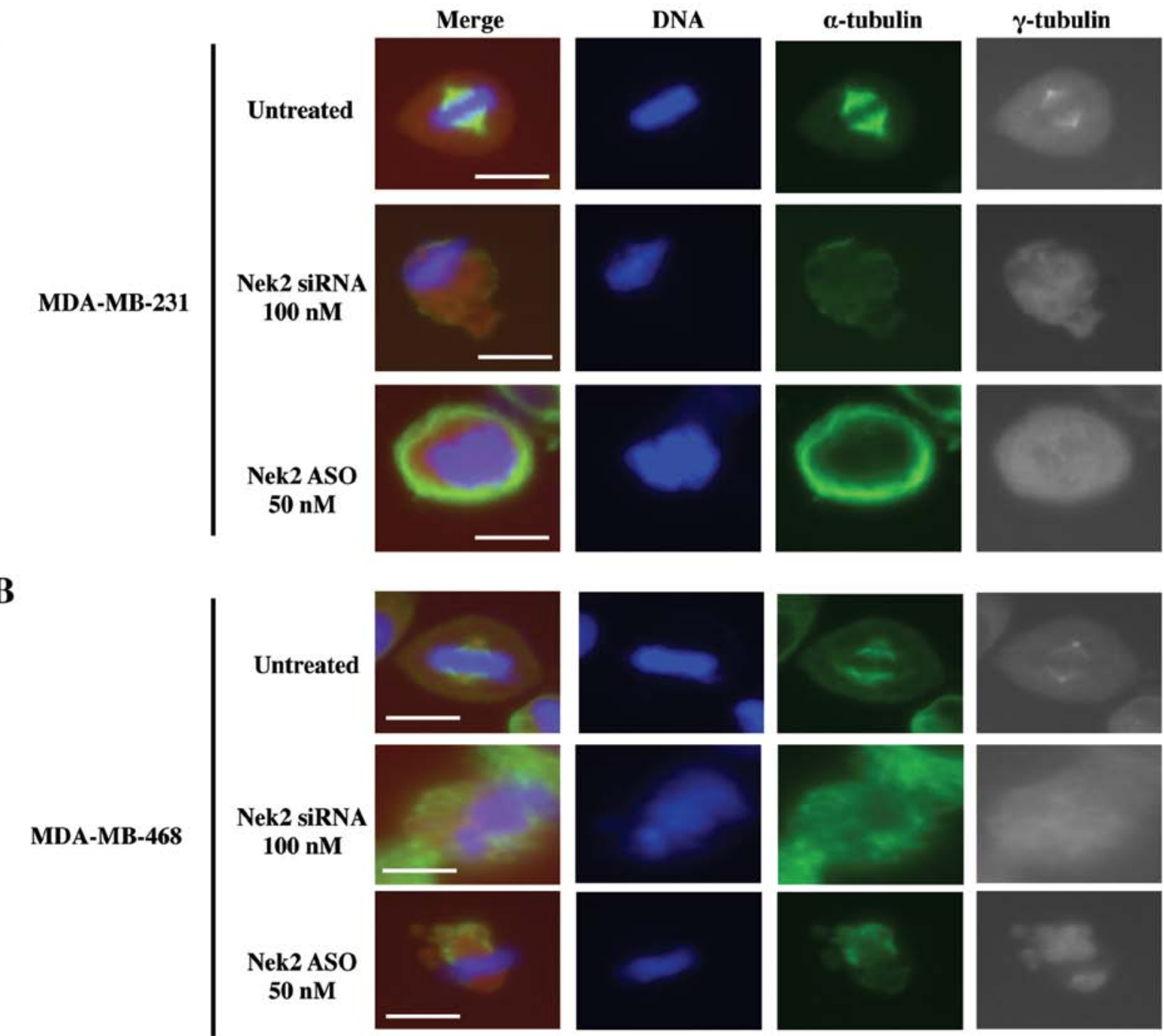

Figure 4. Disruption of spindles and spindle fiber formation after introduction of Nek2 siRNA or ASO. Immunofluorescence image of ASO or siRNA transfected (A) M231 and (B) M468 breast cancer cells. Both cell lines were transfected with Nek2 siRNA or ASO before cell cycle synchronization with thymidine. Synchronized cells were either untreated or transfected with Nek2 $100 \mathrm{nM}$ siRNA or $50 \mathrm{nM}$ ASO, fixed in cold methanol, and stained with antibodies against $\gamma$-tubulin (gray image and red spot in merge) and $\alpha$-tubulin (green). DNA was counterstained with DAPI (blue) (magnification; bar, $50 \mu \mathrm{m}$ ). Both Nek2 depleted cell lines demonstrated diffused spindle poles, unusual microtubule formation, weak $\alpha$-tubulin staining, and misaligned chromosomes. The results are representative of 3 independent trials.

lines were observed. These abnormal mitotic structures were classified into several types. First, Nek2 downregulated cells retained fewer microtubules (Fig. 4A and B, green images), and the $\gamma$-tubulin centrosome-associated signal was weak (Fig. 4A and B, gray images) compared to untreated cells. Lack of clear centrosomal staining against $\gamma$-tubulin suggests it is lost due to Nek2 depletion. Alternatively, duplicated centrosomes were not separated properly, or microtubule formation from the recently separated centrosomes was defective. Also, because Nek 2 regulates chromosome alignment and signaling of the spindle assembly checkpoint (26), inhibition of Nek2 by siRNA or ASO lead to misaligned chromosomes at metaphase (Fig. 4A and B, DAPI staining). This data suggests that Nek2 is required for proper mitotic spindle formation since cell death ensued as a result of abnormal microtubule generation or centrosome duplication/separation upon Nek2 silencing with either siRNA or ASO.
The effects of Nek2 siRNA or ASO transfection on cell cycle distribution and apoptosis. Before studying combinatorial treatment efficacy for siRNA and ASO with anticancer drugs, it was important to establish whether cell cycle distribution and apoptosis levels would be changed with Nek2 depletion.

siRNA or ASO treated M231 cell lines showed little mitotic activity (Fig. 5). While a small increase in the percent of cells in $4 n$ was observed regardless of treatment, significant changes in cell cycle profiles between control and siRNA or ASO transfected cells was not evident. Treatment with $100 \mathrm{nM}$ siRNA increased cells in $4 \mathrm{n}$ by $10 \%$. Cells treated with $50 \mathrm{nM}$ ASO demonstrated a $6 \%$ increase in $4 \mathrm{n}$ compared to controls (Fig. 5A).

To determine whether Nek2 gene silencing alone caused apoptosis and how apoptosis induction compared to a commonly used anticancer drug for TNBC, cells were analyzed by FACS $24 \mathrm{~h}$ after siRNA/ASO transfection and paclitaxel treatment. M231 cells treated with $1 \mu \mathrm{M}$ paclitaxel alone, showed $41 \%$ 
A

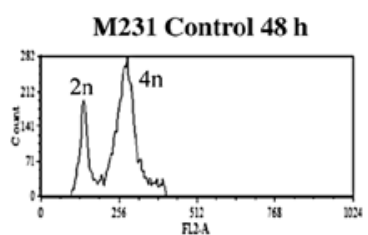

M231 100 nM SIRNA 48 h

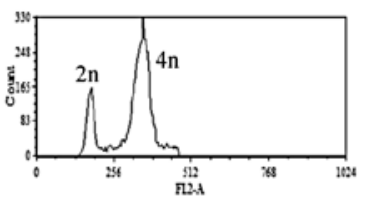

M231 50 nM ASO $48 \mathrm{~h}$

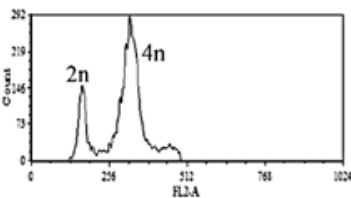

B
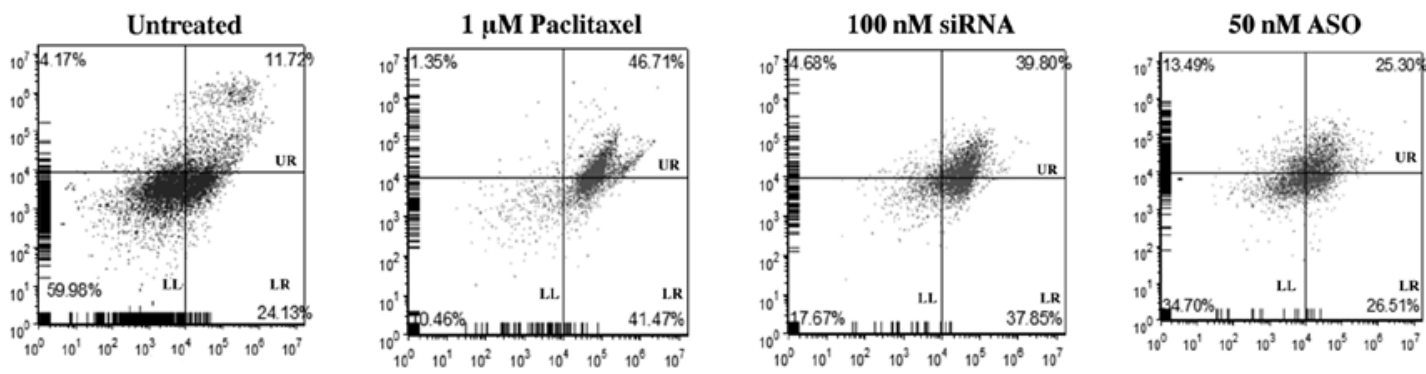

PI

Annexin V-FITC

C

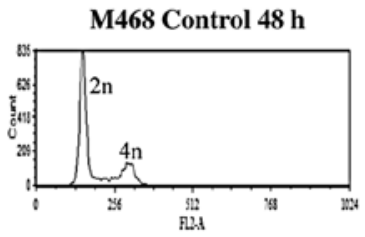

M468 $100 \mathrm{nM}$ siRNA $48 \mathrm{~h}$

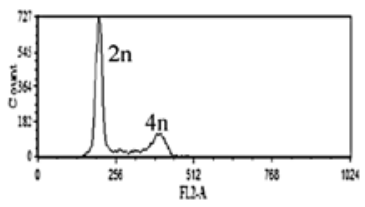

MD468 $50 \mathrm{nM}$ ASO $48 \mathrm{~h}$

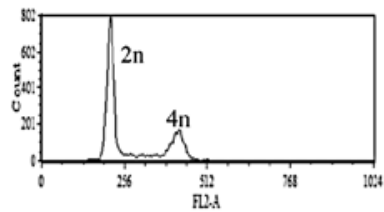

D

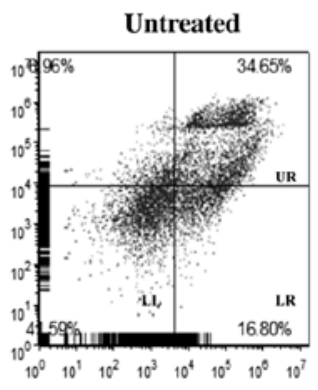

$1 \mu \mathrm{M}$ Paclitaxel
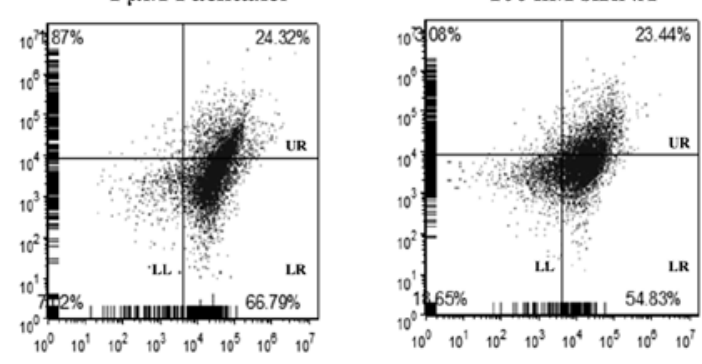

50 nM ASO

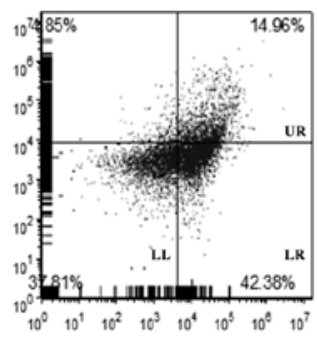

PI

Annexin V-FITC

Figure 5. Representative results of cell cycle distribution and apoptosis for $100 \mathrm{nM}$ Nek2 siRNA or $50 \mathrm{nM}$ Nek2 ASO in M468 and M231 TNBC cells. (A) M231 and (C) M468 cell cycle distribution of non-transfected (left), $100 \mathrm{nM}$ siRNA (middle), or $50 \mathrm{nM}$ ASO (right). Cell cycle was synchronized using a double thymidine block. Time indicates released time after thymidine treatment. After cell cycle synchronization, cells were transfected with siRNA or ASO. Cells were harvested $48 \mathrm{~h}$ later. Cells were fixed in ethanol and the DNA content was analyzed by flow cytometry following propidium iodide (PI) staining. In A and C, the x-axis demonstrates fluorescence intensity based on DNA content, and the y-axis corresponds to the number of fluorescent cells. B and D represent parallel cell cultures stained using FITC-conjugated Annexin V and PI to analyze apoptosis using flow cytometry (LL, live cells; LR, apoptotic cells; UR, dead, necrotic and late apoptotic cells). Cells were treated with $1 \mu \mathrm{M}$ paclitaxel, $100 \mathrm{nM}$ siRNA or $50 \mathrm{nM}$ ASO, respectively. Data are representative results from at least 3 independent experiments demonstrating reproducibility.

increase in cell death. Similarly, $37 \%$ increase in cell death was observed with $100 \mathrm{nM}$ siRNA alone. Transfection of $50 \mathrm{nM}$ ASO showed no appreciable difference in cell death (26\%), over untreated cells (24\%) (Fig. 5B).

M468 cell treatments demonstrated similar cell cycle distribution results. There was a $12 \%$ accumulation in 4 n DNA content for control cells but only $17 \%$ for siRNA and $15 \%$ for ASO-transfected cells (Fig. 5C). However, M468 cells were more sensitive to apoptosis induction. Paclitaxel $(1 \mu \mathrm{M})$ induced apoptosis in $66 \%$ of the treated cell population. siRNA $(100 \mathrm{nM})$ increased apoptosis to 54\%. Interestingly, $50 \mathrm{nM}$ ASO treatment increased apoptosis to $42 \%$ compared to the $16 \%$ observed for controls.
Combinatorial anticancer drug treatment effects on triple negative breast cancer cells. In order to address this question, paclitaxel and doxorubicin were chosen because they work through different mechanisms of action. Paclitaxel binds to the tubulin heterodimer, effectively stabilizing microtubules by inhibiting depolymerization, resulting in transient arrest in mitosis, development of a multinucleated interphase, followed by apoptosis $(27,28)$. Doxorubicin inhibits release of DNA torsion during replication and transcription by immobilizing the topoisomerase II-DNA complex (29-31).

The effects of combinatorial treatment on M231 cells. To determine whether M231 cell sensitivity to paclitaxel was augmented 
A

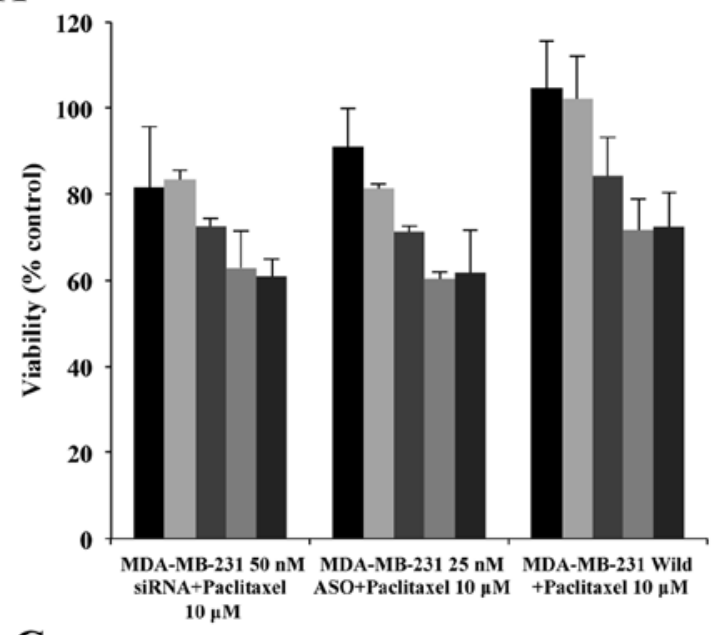

C

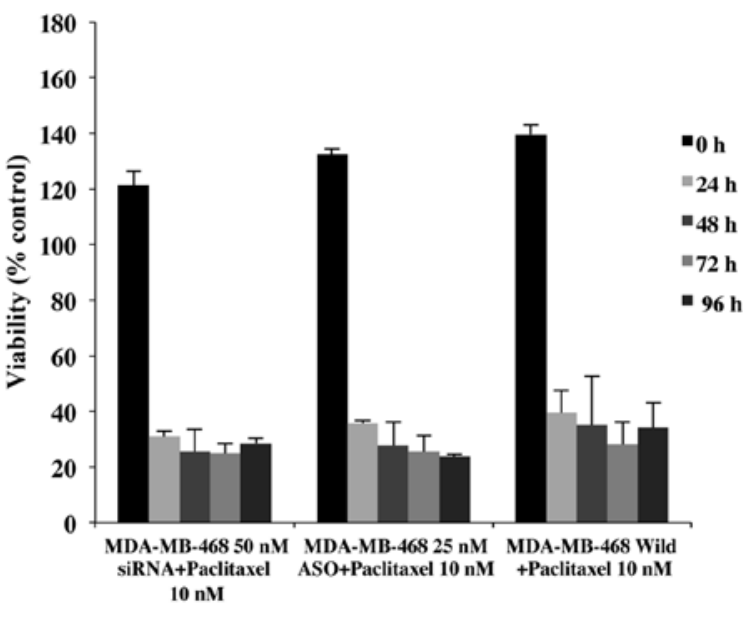

B

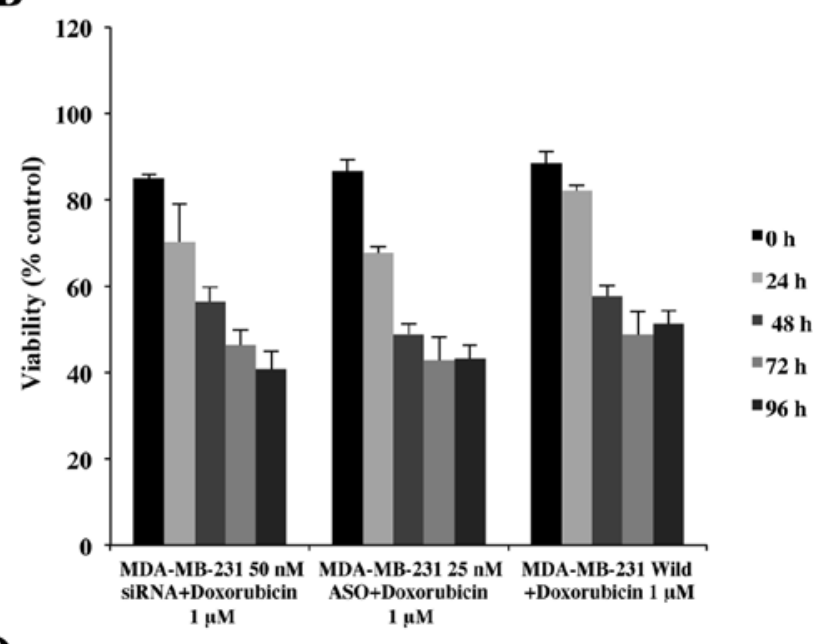

D

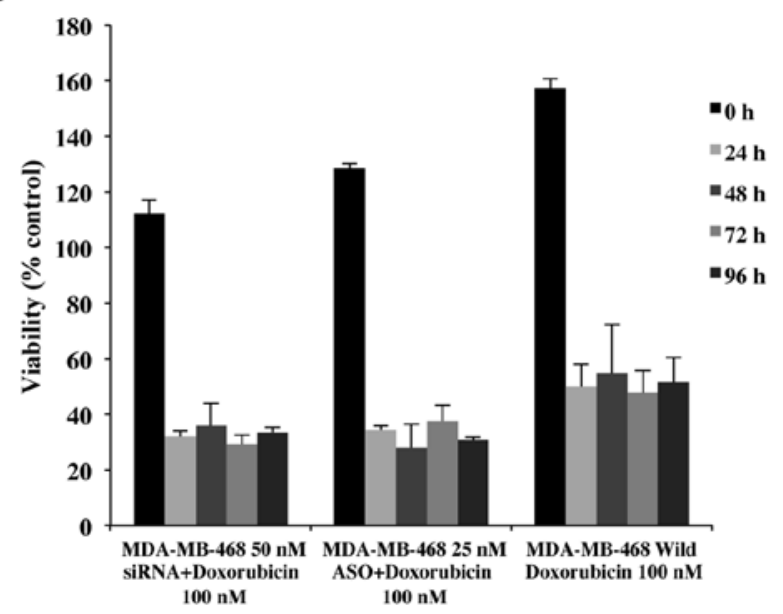

Figure 6. Effects of Nek2 siRNA or ASO in combination with paclitaxel or doxorubicin on the cell viability of M468 and M231 TNBC cell lines. Cell viability was determined using the XTT assay after 10-h incubation. For this study, cell viability was standardized to viability of non-transfected/untreated cells at 0 h. (A and B) Viability of $50 \mathrm{nM}$ siRNA, $25 \mathrm{nM}$ ASO transfected and non-transfected M231 cells with (A) $10 \mu \mathrm{M}$ of paclitaxel treatment or (B) $1 \mu \mathrm{M}$ of doxorubicin. (C and D) Viability of $50 \mathrm{nM}$ siRNA, $25 \mathrm{nM}$ ASO transfected and non-transfected M468 cells with (C) $10 \mathrm{nM}$ of paclitaxel treatment or (D) $100 \mathrm{nM}$ of doxorubicin. These graphs represent the results from the combinatorial study performed in triplicate from at least 3 independent experiments (error bar, standard deviations); $0 \mathrm{~h}$ indicates initial time point immediately after cells were treated with anticancer drug only or anticancer drug and + siRNA or ASO.

with this combinatorial approach, concentrations from $10 \mathrm{nM}$ to $10 \mu \mathrm{M}$ of paclitaxel were added to $\mathrm{M} 231$ cells pretreated with $50 \mathrm{nM}$ siRNA or $25 \mathrm{nM}$ ASO (Fig. 6A). Although cell viability with $50 \mathrm{nM}$ siRNA + paclitaxel treated cells showed levels below paclitaxel only treated cells, there was no significant difference between siRNA combinatorial treatment and paclitaxel concentrations up to $1 \mu \mathrm{M}$ (data not shown). However, as demonstrated in Fig. 6A, significant decreases in cell viability (60\% vs. $72 \%$ ) were observed in combination with $10 \mu \mathrm{M}$ paclitaxel. Additionally, this combination of siRNA and paclitaxel generated a consistent decrease through $96 \mathrm{~h}$ whereas other combinations showed cell recovery at 72 or $96 \mathrm{~h}$.

Interestingly, $25 \mathrm{nM}$ ASO transfected M231 cells, in combination with low concentrations of paclitaxel $(10,100 \mathrm{nM}$ and $1 \mu \mathrm{M}$ paclitaxel), demonstrated decreases in cell viability from 13 to $30 \%$ (data not shown), comparable to $50 \mathrm{nM}$ siRNA $+10 \mu \mathrm{M}$ paclitaxel (Fig. 6A). Cell viability for $25 \mathrm{nM}$ $\mathrm{ASO}+10 \mu \mathrm{M}$ paclitaxel was reduced by 19 to $29 \%$ (Fig. 6A) compared to controls. These data confirmed that Nek2 silenced by siRNA or ASO, increased M231 cell sensitivity to paclitaxel resulting in overall decreased cell viability.

Cells treated with doxorubicin in combination with siRNA or ASO against Nek2 demonstrated variable results. M231 cells treated with $10 \mathrm{nM}$ doxorubicin alone showed no significant changes in cell viability until $96 \mathrm{~h}$ at which time, cell viability increased significantly. Cells treated with $50 \mathrm{nM}$ siRNA or $25 \mathrm{nM}$ ASO in combination with $10 \mathrm{nM}$ doxorubicin showed significant decreased cell viability until 72 h. At 96 h cells treated with siRNA + doxorubicin recovered slightly, whereas ASO-doxorubicin mirrored the cell viability increase observed in doxorubicin alone. Similar trends were observed for combinations of siRNA or ASO with $100 \mathrm{nM}$ doxorubicin.

Comparable cell viability effects were observed with $50 \mathrm{nM}$ siRNA and $25 \mathrm{nM}$ ASO transfected cells $+1 \mu \mathrm{M}$ doxorubicin (Fig. 6B) and $10 \mu \mathrm{M}$ of doxorubicin (data not shown). Cell viabilities continued to decrease through the end-point of the experiment. In summary, although 10 and $100 \mathrm{nM}$ doxorubicin + siRNA or ASO appeared to increase cell viability during 
the culture period, the effects of 1 and $10 \mu \mathrm{M}$ doxorubicin on M231 cells were significantly enhanced with the addition of Nek2 silencing by siRNA or ASO.

The effects of combinatorial treatment on M468 cells. Control cells treated with $10 \mathrm{nM}$ to $10 \mu \mathrm{M}$ of paclitaxel, all showed increased viabilities at $96 \mathrm{~h}$ of treatment. However, even though the viability levels of transfected M468 cells were not significantly different between siRNA and ASO transfected cells with various concentrations of paclitaxel treatment, all of the cell viabilities of transfected cells were lower than non-transfected cells and remained at similar levels or showed minor increases at $96 \mathrm{~h}$ (Fig. 6C). In general, paclitaxel treatment in combination with $50 \mathrm{nM}$ siRNA or $25 \mathrm{nM}$ ASO did not significantly decrease viability compared with paclitaxel alone.

Doxorubicin treatment in combination with Nek2 inhibition had a more profound effect in M468 cells than M231 cells. No significant increase in cell viability was observed for control cells treated with $100 \mathrm{nM}, 1$ or $10 \mu \mathrm{M}$ doxorubicin. M468 cells transfected with siRNA or ASO resulted in significantly decreased cell viability (Fig. 6D). Cells transfected with $50 \mathrm{nM}$ siRNA or with $25 \mathrm{nM}$ ASO plus $100 \mathrm{nM}$ doxorubicin showed $>34 \%$ viability. In contrast, cells in doxorubicin alone showed $\sim 50 \%$ viability (Fig. 6D). These results indicate that a combinatorial approach of Nek2 gene silencing with siRNA or ASO and an anticancer drug increased M468 cell sensitivity compared to anticancer drug administration alone.

Interestingly, we observed fluctuations in cell viability with $10 \mathrm{nM}$ doxorubicin $+50 \mathrm{nM}$ siRNA or $25 \mathrm{nM}$ ASO. After $24 \mathrm{~h}$, cell viability for Nek2 silenced cells + doxorubicin was 36 and $43 \%$, respectively. In $10 \mathrm{nM}$ doxorubicin treatment alone, cell viability was $53 \%$. After $24 \mathrm{~h}$ cell viability increased by $20 \%$ and after $72 \mathrm{~h}$ an additional $40 \%$ increase was observed. Although siRNA and ASO + doxorubicin showed slight increases in cell viability, the overall increases compared to non-transfected, at $96 \mathrm{~h}$, doxorubicin controls were $\sim 70 \%$ lower than controls.

\section{Discussion}

In this study, we investigated Nek2 as a potential drug target in cancer treatment. Additionally, we were interested in whether pre-conditioning the cells through Nek2 silencing would augment the effectiveness of current anticancer drugs and in determining whether a combinatorial approach would maintain treatment effectiveness while decreasing anticancer drug concentrations, potentially decreasing associated side-effects. In order to address these questions, we utilized two well-characterized TNBC cell lines (MDA-MB-231 and MDA-MB-468).

When comparing the effects of Nek2 depletion by siRNA or ASO on cell viability, we observed that overall, ASO treated cells exhibited lower cell viability than siRNA treated cells. Indeed, the efficiency of siRNA and ASO is controversial. Some studies reported that when compared, siRNA was more efficient and its effect was longer than ASO $(32,33)$. In contrast, Tsui et al (2005) described that the efficiency of siRNA was comparable to ASO (34). Our cell viability results suggest that the effectiveness of siRNA and ASO may be cell type dependent. Alternatively, efficiency may depend upon the target gene.
Before depleting Nek2 using siRNA and ASO, we predicted that cell cycle would be arrested in the G2/M phase based on other cell cycle kinase studies (33). Although it has been previously shown that Nek 2 is one of the cell cycle kinases (18-24), our results showed that Nek2 gene silencing did not induce strong mitotic arrest at G2/M phase, but instead induced apoptosis, indicating that the role of Nek2 may be different from other cell cycle-related kinases in its regulation of cell cycle. Additionally, inactive Nek2A in human cells did not block cell cycle progression (21). It may be possible that other Nek2 family members (i.e., Nek1 to Nek11), or other cell cycle kinases such as Plk1 or Aurora A compensate for the loss of Nek2 function. However, Nek2 depleted cells have been reported to have abnormal mitotic characteristics induced through several different categories. First, Nek2 depletion interferes with centrosome duplication or maturation and arrangement of proteins including $\gamma$-tubulin, Plk1 and nucleophosmin/B23 onto the mitotic spindle poles. Secondly, Nek2 silencing induces abnormal chromosome segregation in human cells (35). Thirdly, Nek2 depletion interferes with the regulation of centrosome separation. Finally, depletion of Nek2 causes arrest of cell proliferation and increases apoptosis as a result of mitotic errors (36). As we suspected, Nek2 depletion affected the kinetochores-microtubule attachment, inducing a spindle checkpoint imbalance and abnormal clustering of kinetochore components, resulting in increased sensitivity of cells to the microtubule targeting anticancer drug paclitaxel.

The increase in cell sensitivity of Nek2-depleted TNBC cells in combination with doxorubicin may be due to the downregulation of TRF1 and the checkpoint kinase, Chk2, inducing chromosomal abnormalities and altering the cell cycle (31). Prime et al demonstrated interactions between Trf1 with Mad1 and Nek2 (37). However, more study is needed to elucidate their relationship with Nek2 and the implications for cancer development.

Our results suggest that combinational administration of Nek2 depletion and these chemotherapy agents increase TNBC cell sensitivity to anticancer treatment. We observed that the effects of siRNA and ASO + anticancer agent was comparable for both cells and showed significant decreases from control non-transfected cells treated with agent alone. By pretreating the patient with siRNA or ASO therapies, the potential exists for equivalent or higher sensitization with lower dosages anticancer drugs. While achieving the same overall effect, the side-effects to the patient may be reduced.

\section{Acknowledgements}

We wish to thank the TTU Imaging Center, the TTU Biotechnology Core Facilities as well as Dr Dmitri Pappas (Department of Chemistry and Biochemistry) for access to the FACSCalibur cell sorter.

\section{References}

1. Baselga J, Norton L, Albanell J, Kim YM and Mendelsohn J: Recombinant humanized anti-HER 2 antibody (Herceptin) enhances the antitumor activity of paclitaxel and doxorubicin against HER2/neu overexpressing human breast cancer xenografts. Cancer Res 58: 2825-2831, 1998.

2. Cleator S, Heller W and Coombes RC: Triple-negative breast cancer: therapeutic options. Lancet Oncol 8: 235-244, 2007. 
3. Rakha EA and Ellis IO: Triple-negative/basal-like breast cancer: review. Pathology 41: 40-47, 2009.

4. Gluz O, Liedtke C, Gottschalk N, Pusztai L, Nitz U and Harbeck N: Triple-negative breast cancer - current status and future directions. Ann Oncol 20: 1913-1927, 2009.

5. Carey L, Winer E, Viale G, Cameron D and Gianni L: Triple-negative breast cancer: disease entity or title of convenience? Nat Rev Clin Oncol 7: 683-692, 2010.

6. Pérez de Castro I, de Cárcer G, Montoya G and Malumbres M: Emerging cancer therapeutic opportunities by inhibiting mitotic kinases. Curr Opin Pharmacol 8: 375-383, 2008.

7. Chi YH and Jeang KT: Aneuploidy and cancer. J Cell Biochem 102: 531-538, 2007.

8. Kops GJ, Weaver BA and Cleveland DW: On the road to cancer: aneuploidy and the mitotic checkpoint. Nat Rev Cancer 5: 773-785, 2005

9. Yuen KW, Montpetit B and Hieter P: The kinetochore and cancer: what's the connection? Curr Opin Cell Biol 17: 576-582, 2005.

10. Fry AM, Mayor T and Nigg EA: Regulating centrosomes by protein phosphorylation. Curr Top Dev Biol 49: 291-312, 2000.

11. Nigg EA: Mitotic kinases as regulators of cell division and its checkpoints. Nat Rev Mol Cell Biol 2: 21-32, 2001

12. Takai N, Miyazaki T, Fujisawa K, Nasu K, Hamanaka R and Miyakawa I: Polo-like kinase (PLK) expression in endometrial carcinoma. Cancer Lett 169: 41-49, 2001.

13. Holtrich U, Wolf G, Bräuninger A, et al: Induction and down-regulation of PLK, a human serine/threonine kinase expressed in proliferating cells and tumors. Proc Natl Acad Sci USA 91: 1736-1740, 1994.

14. Li D, Zhu J, Firozi PF, et al: Overexpression of oncogenic STK15/BTAK/Aurora A kinase in human pancreatic cancer. Clin Cancer Res 9: 991-997, 2003.

15. Bettencourt-Dias M and Glover DM: Centrosome biogenesis and function: centrosomics brings new understanding. Nat Rev Mol Cell Biol 8: 451-463, 2007.

16. Krämer A, Neben K and Ho AD: Centrosome aberrations in hematological malignancies. Cell Biol Int 29: 375-383, 2005.

17. Nigg EA: Centrosome aberrations: cause or consequence of cancer progression? Nat Rev Cancer 2: 815-825, 2002

18. Schultz SJ, Fry AM, Sütterlin C, Ried T and Nigg EA Cell cycle-dependent expression of Nek2, a novel human protein kinase related to the NIMA mitotic regulator of Aspergillus nidulans. Cell Growth Differ 5: 625-635, 1994.

19. Fry AM, Schultz SJ, Bartek J and Nigg EA: Substrate specificity and cell cycle regulation of the Nek2 protein kinase, a potential human homolog of the mitotic regulator NIMA of Aspergillus nidulans. J Biol Chem 270: 12899-12905, 1995.

20. Fry AM, Meraldi P and Nigg EA: A centrosomal function for the human Nek2 protein kinase, a member of the NIMA family of cell cycle regulators. EMBO J 17: 470-481, 1998.

21. Faragher AJ and Fry AM: Nek2A kinase stimulates centrosome disjunction and is required for formation of bipolar mitotic spindles. Mol Biol Cell 14: 2876-2889, 2003.
22. Hayward DG, Clarke RB, Faragher AJ, Pillai MR, Hagan IM and Fry AM: The centrosomal kinase Nek2 displays elevated levels of protein expression in human breast cancer. Cancer Res 64: 7370-7376, 2004.

23. Tsunoda N, Kokuryo T, Oda K, et al: Nek2 as a novel molecular target for the treatment of breast carcinoma. Cancer Sci 100: 111-116, 2009.

24. Kokuryo T, Senga T, Yokoyama Y, Nagino M, Nimura Y and Hamaguchi M: Nek2 as an effective target for inhibition of tumorigenic growth and peritoneal dissemination of cholangiocarcinoma. Cancer Res 67: 9637-9642, 2007.

25. Crown $J$ and Pegram M: Platinum-taxane combinations in metastatic breast cancer: an evolving role in the era of molecularly targeted therapy. Breast Cancer Res Treat 79 (Suppl 1): S11-S18, 2003

26. Wei R, Ngo B, Wu G and Lee WH: Phosphorylation of the Ndc80 complex protein, HEC1, by Nek2 kinase modulates chromosome alignment and signaling of the spindle assembly checkpoint. Mol Biol Cell 22: 3584-3594, 2011.

27. Jordan MA and Wilson L: Microtubules as a target for anticancer drugs. Nat Rev Cancer 4: 253-265, 2004.

28. Blajeski AL, Kottke TJ and Kaufmann SH: A multistep model for paclitaxel-induced apoptosis in human breast cancer cell lines. Exp Cell Res 270: 277-288, 2001.

29. Isaacs RJ, Davies SL, Sandri MI, Redwood C, Wells NJ and Hickson ID: Physiological regulation of eukaryotic topoisomerase II. Biochim Biophys Acta 1400: 121-137, 1998.

30. Nitiss JL and Beck WT: Antitopoisomerase drug action and resistance. Eur J Cancer 32A: 958-966, 1996.

31. Spallarossa P, Altieri P, Aloi C, et al: Doxorubicin induces senescence or apoptosis in rat neonatal cardiomyocytes by regulating the expression levels of the telomere binding factors 1 and 2 . Am J Physiol Heart Circ Physiol 297: H2169-H2181, 2009.

32. Bertrand JR, Pottier M, Vekris A, Opolon P, Maksimenko A and Malvy C: Comparison of antisense oligonucleotides and siRNAs in cell culture and in vivo. Biochem Biophys Res Commun 296: 1000-1004, 2002.

33. Spänkuch-Schmitt B, Bereiter-Hahn J, Kaufmann M and Strebhardt K: Effect of RNA silencing of polo-like kinase-1 (PLK1) on apoptosis and spindle formation in human cancer cells. J Natl Cancer Inst 94: 1863-1877, 2002.

34. Tsui P, Rubenstein M and Guinan P: siRNA is not more effective than a first generation antisense oligonucleotide when directed against EGFR in the treatment of PC-3 prostate cancer. In Vivo 19: $653-656,2005$.

35. Lou Y, Yao J, Zereshki A, et al: NEK2A interacts with MAD1 and possibly functions as a novel integrator of the spindle checkpoint signaling. J Biol Chem 279: 20049-20057, 2004.

36. Fletcher L, Cerniglia GJ, Yen TJ and Muschel RJ: Live cell imaging reveals distinct roles in cell cycle regulation for Nek2A and Nek2B. Biochim Biophys Acta 1744: 89-92, 2005.

37. Prime $G$ and Markie D: The telomere repeat binding protein Trf1 interacts with the spindle checkpoint protein Mad1 and Nek2 mitotic kinase. Cell Cycle 4: 121-124, 2005. 